\title{
2D and 3D pore structure characterization of bi-layered porous polyethylene barrier membrane using SEM and micro-CT
}

\author{
Ji-Chuan Song $^{\mathrm{a}, \mathrm{b}}$, Jintamai Suwanprateeb ${ }^{\mathrm{c}, *}$, Daraporn Sae-lee ${ }^{\mathrm{b}, \mathrm{d}}$, Teerapan Sosakul ${ }^{\mathrm{d}}$, \\ Waranuch Pitiphat ${ }^{\mathrm{e}, \mathrm{f}}$, Saengsome Prajaneh ${ }^{\mathrm{f}, \mathrm{g}}$, Suwadee Kositbowornchai ${ }^{\mathrm{g}}$, Boonsong Putraphan ${ }^{\mathrm{h}}$ \\ a Doctoral Program in Oral Science, Faculty of Dentistry, Khon Kaen University, \\ Khon Kaen 40002 Thailand \\ b Neuroscience Research and Development Group, Khon Kaen University, Khon Kaen 40002 Thailand \\ c Biofunctional Materials and Devices Research Group, \\ National Metal and Materials Technology Centre (MTEC), Pathumthani 12120 Thailand \\ d Department of Prosthodontics, Faculty of Dentistry, Khon Kaen University, Khon Kaen 40002 Thailand \\ e Department of Preventive Dentistry, Faculty of Dentistry, Khon Kaen University, \\ Khon Kaen 40002 Thailand \\ ${ }^{f}$ Research Group of Chronic Inflammatory Oral Diseases and Systemic Diseases Associ- \\ ated with Oral Health, Khon Kaen University, \\ Khon Kaen 40002 Thailand \\ g Department of Oral Biomedical Science, Faculty of Dentistry, Khon Kaen University, \\ Khon Kaen 40002 Thailand \\ h Oral Biology Research Centre, Faculty of Dentistry, Chulalongkorn University, Bangkok 10330 Thailand \\ *Corresponding author, e-mail: jintamai@mtec.or.th
}

Received 26 May 2018

Accepted 30 Apr 2019

\begin{abstract}
Barrier membrane is an essential component in guided bone regeneration for successful bone augmentation in implant dentistry. The microstructure of barrier membrane can remarkably impact its mechanical properties and biological performances. This study was aimed to investigate the pore structures of a bi-layered porous polyethylene (PPE) barrier membrane by 2D and 3D characterization techniques. Two opposite sides of PPE barrier membrane were imaged with scanning electron microscope and micro-computed tomography (micro-CT). The 2D and 3D pore characteristics were then analysed with associated software, respectively. Both techniques similarly showed that PPE barrier membrane comprised two different structures including one with low porosity (smooth) and one with high porosity (coarse) as designed. In 2D analysis, both surfaces possessed similar positively skewed distributions in pore area and circle diameter. The smooth side had significantly smaller pore amount, pore density, surface porosity, pore area, circle diameter, Feret diameter and aspect ratio, but larger roundness, circularity and solidity than the coarse side $(p<0.05)$. In 3D analysis, the smooth side possessed significantly smaller pore diameter and volume porosity than the coarse one $(p<0.05)$. No significant differences in strut thickness, specific surface area, connectivity density (Conn.D), and degree of anisotropy (DA) were found between two layers $(p>0.05)$. The combination of 2D and 3D techniques could be effectively employed to characterize the pore microstructure and morphology of PPE barrier membrane. The limitations of each technique were also discussed.
\end{abstract}

KEYWORDS: bi-layered porous polyethylene membrane, guided bone regeneration, scanning electron microscopy, micro-computed tomography, image analysis

\section{INTRODUCTION}

Partial and total edentulisms are common dysfunctions following tooth extraction and loss in dental practice. Conventional fixed and removable dentures usually cannot preserve the alveolar bone volume due to the lack of internal loading, or may even accelerate the bone resorption process for inappropriate prosthetic loading ${ }^{1}$. The sequelae will be narrow and flat alveolar ridges ${ }^{2}$. These atrophic ridges present huge challenges to ideal dental implant placement and restoration, thus impairing 
implant function, prosthesis aesthetics, long-term implant survival and success.

To repair the alveolar defects for ideal implant placement and rehabilitation, multiple bone reconstruction strategies have been intensively investigated. Although no consensus exists on which one is the most efficient, the guided bone regeneration (GBR) technique is reported as a predictable one in various defect scenarios (e.g., implant fenestration, dehiscence, and even segmental defects) ${ }^{3}$. It can efficiently regenerate the lost bone tissue with the synergistic application of barrier membrane and bone substitute.

In this technique, barrier membrane not only mechanically protects blood clot, stabilizes bone substitutes, creates and maintains space for new bone formation within defects, but also biologically isolates the fast-growing fibroblasts and connective tissue from premature penetration into the created space. Currently, various natural and synthetic barrier materials, including bioabsorbable (e.g., collagen, chitosan, alginate, aliphatic polyesters and their co-polymers) and nonabsorbable (e.g., titanium, expanded/dense polytetrafluoroethylene), have been extensively investigated for clinical applications, alone or in combination ${ }^{4,5}$. Although some disadvantages (e.g., premature wound dehiscence, membrane exposure) remain, expanded polytetrafluoroethylene (e-PTFE) membrane is considered as the gold standard for its superior space maintenance and predictable bone regeneration potential, especially for the intractable large and vertical alveolar defects. Besides the biocompatibility and tissue integration, a barrier membrane should basically possess appropriate cell occlusiveness, space maintenance and clinical maneuverability as well ${ }^{4,5}$. The layered barrier membrane with differential pore sizes and porosities is believed to be a future trend for mimicking bone structures and providing suitable mechanical properties ${ }^{5,6}$. The acquisition of these above properties is closely associated with the two- and three-dimensional microstructural characteristics of both pores and matrix of the membranes, such as pore size, pore size distribution (PSD), pore geometry, pore distribution, porosity, interconnectivity, strut thickness, connectivity density (Conn.D) and degree of anisotropy (DA). Hence accurate characterization of these features is very helpful to evaluate the membrane's mechanical properties and biological performances.

To date, a variety of techniques have been proposed for characterizing porous microarchitectures of bones, scaffolds and other biomaterials, including gravimetry, liquid displacement, permeability-based method, mercury intrusion porosimetry (MIP), histology, gas pycnometry, gas adsorption, scanning electron microscopy (SEM) and confocal microscopy, etc. However, there is currently no wellrecognized standardization technique for architectural characterization. They each have specific indications, advantages and disadvantages ${ }^{7,8}$. For instance, MIP is reliable for porous structure quantification, but the stress sensitivity, toxicity and high cost limit its wide use, especially in compressible biomaterials. SEM can enable topological surface characterization, but limits to provide 2D information. Others may present their own disadvantages, such as low accuracy, technique sensitivity, timeconsuming sample preparation, sample destruction and high cost. With the rapid development of advanced medical imaging, image analysis and computer science, micro-computed tomography (microCT) has been proposed to quantify the intricate internal microarchitectures of bone, cardiovascular tissues, scaffolds and other biomaterials for its unparalleled advantages ${ }^{9-11}$. It can achieve quick nondestructive three-dimensional imaging, reconstruction, segmentation, visualization and quantification under the aid of sophisticated software without prior sample preparation ${ }^{7-11}$. It can even simulate the micromechanics by combining with finite element method ${ }^{12,13}$.

Polyethylene is one of the most commonly-used polymeric biomaterials. It possesses good physicochemical, mechanical and biological properties, including bioinertness, volume stability, flexibility, fatigue resistance, mechanical strength, and clinical maneuverability ${ }^{14,15}$. When fabricated as porous form, porous polyethylene did not only demonstrate biocompatibility, but also permitted fibrovascularization and bone ingrowth ${ }^{16,17}$, which offered advantages over non-porous implants in craniofacial, oral and maxillofacial and plastic surgeries for tissue repair and augmentation ${ }^{17,18}$. It was also employed as bone graft containment for alveolar ridge preservation ${ }^{19}$. It was anticipated that porous polyethylene could be applied as a barrier membrane in GBR for repairing the intractable large and/or vertical alveolar defects. A bi-layered porous polyethylene (PPE) barrier membrane which contained both lowand high-porosity sides in a single membrane was, thus conceived and developed. This was based on the formulation of porous polyethylene that was previously developed by our group ${ }^{20,21}$. In clinical practice, the low-porosity surface would be placed facing the mucoperiosteal flap for inhibiting prema- 
ture soft tissue penetration, while the high-porosity one facing the bone defect for encouraging bone ingrowth.

The aim of this study was to investigate the pore structure characteristics within two sides of the bi-layered PPE barrier membrane by using both 2D and 3D characterization techniques. The results were anticipated to provide preliminary evidence to validate the above assumptions, and to assess the biological responses of this membrane in clinical practice.

\section{MATERIALS AND METHODS}

\section{Bi-layered PPE membrane preparation}

Raw materials used were high-density polyethylene (Thaizex 7000F, Bangkok Polyethylene Co., Thailand), maltodextrin (Shandong Duqing, Inc., China) and polyvinyl alcohol (Sigma-Aldrich, USA). They were mixed at a ratio of 70:20:10 w/w. Prior to mixing, high-density polyethylene was grinded into powders with a particle size of approximately $305 \mu \mathrm{m}$ determined by a Mastersizer (Malvern Instruments, UK). Other raw materials were supplied in powder with particle size ranging $80-100 \mu \mathrm{m}$, and used without further sieving. Bi-layered porous polyethylene membrane specimens were fabricated by a single step moulding technique. Briefly, $0.7 \mathrm{~g}$ of mixture was loaded into a rectangular mould with a cavity of $60 \times 40 \times 0.3 \mathrm{~mm}^{3}$, pressurized to produce a differential pressure across the thickness of the mould, and heated at $145^{\circ} \mathrm{C}$ for $45 \mathrm{~min}$ using a wet salt bed technique as reported elsewhere ${ }^{22}$. The mould was left to gradually cool down to room temperature for $2 \mathrm{~h}$. A membrane which contained both low-porosity surface (designated smooth) and high-porosity surface (designated coarse) in single membrane was thus produced. Sample was taken out of the mould and submerged in copious deionized water for $24 \mathrm{~h}$ to leach out binders. Sonication was activated at the first hour to enhance leaching. They were then dried, packaged and sterilized by ethylene oxide gas.

\section{D pore characterization}

Three samples were captured at different zones with a scanning electron microscope (S-3000N, Hitachi, Japan) for each surface at a $\times 40$ magnification, $22.4 \mathrm{~mm}$ working distance and $20.0 \mathrm{kV}$ after gold sputtering. Three typical images were selected for each sample, and loaded in IMAGEJ $1.51 \mathrm{~K}(\mathrm{NIH}$, USA) for topological and geometric analysis. The measurement scale was first set, resulting in a rectangular viewfield of $3200 \times 2400 \mu \mathrm{m}^{2}$ in each image. To achieve optimal image binarization, the segmentation protocol was trialed and established: a duplicate image was first converted into 8-bit greyscale image, and then preprocessed through sequential smoothening, sharpening and contrast enhancement. It was then binarized into black foreground (pores) and white background (polyethylene matrix) by local thresholding. Serial advanced morphological refinement operations, including iterative erosion and dilation, filling holes, and watershed, were performed to achieve as accurate image segmentation as possible by combining image histogram and visual estimation.

To understand additional pore characteristics, a specific pore area cut-off of $7850 \mu \mathrm{m}^{2}$, corresponding to $100 \mu \mathrm{m}$ in diameter of a perfect circle, was set. Hence two ranges of pore area $\left(0 \mu \mathrm{m}^{2}-\right.$ infinity and $7850 \mu \mathrm{m}^{2}$ - infinity) were utilized for parallel 2D pore structure analysis. In IMAGEJ, pores on image edges were excluded for analysis. The circularity was set at $0-1$. All the eligible pores could be checked for fitting with an individual ellipse with equivalent area and perimeter. The pore area, pore amount on each image, surface porosity (area fraction), Feret diameter, aspect ratio of best-fitting ellipse, roundness, circularity, and solidity, could be directly reported. To facilitate the comparison of pore size between two surfaces, all eligible pores were converted into the best-fitting perfect circles with equivalent area. The diameter of the perfect circle, also called circle diameter $(d)$, was calculated as $d=2 \sqrt{\text { area } / \pi}$, where area is the pore area.

To understand the pore distribution on image surface, the pore density was calculated as the ratio of the pore amount on each image reported in IMAGEJ and the area of each image $(3200 \times 2400$ $\mu \mathrm{m}^{2}$ ).

\section{D pore characterization}

PPE specimens $(9.0 \times 9.0 \times 0.3 \mathrm{~mm})$ were mounted in a cylindrical sample holder. They were correctly oriented in the tube centre, and imaged by a microtomographic imaging system ( $\mu$ CT 35, Scanco Medical, Switzerland). Specimens were imaged under $70 \mathrm{kVp}$ and $114 \mu \mathrm{A}$ with a voxel side length of $3 \mu \mathrm{m}$ at a rotation rate of 1000 projections per $180^{\circ}$, and reconstructed with a modified Feldkamp algorithm into 3D models. The integration time was $800 \mathrm{mS}$. Each specimen consisted of approximately $3000 \times 3000 \times 100$ voxels.

A specific software package $\mu$ CT Evaluation Program V 6.5-1 (Scanco Medical, Switzerland) was 


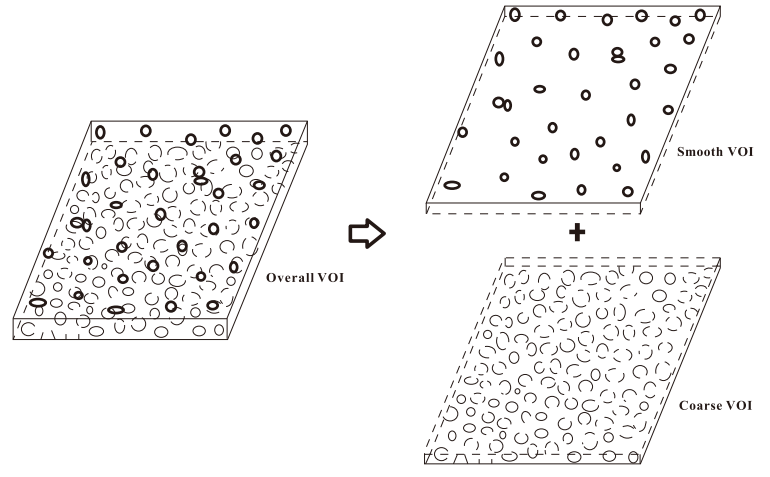

Fig. 1 Schematic of overall, smooth and coarse VOIs virtually defined in micro-CT software. The polymorphous circles in thick and thin lines simply represented the pores on the smooth and coarse surfaces of PPE membrane, respectively. Their dimension, shape and density did not necessarily represent the real pore size, geometry and distribution. The interior pores were not illustrated hereon.

utilized to manipulate the acquired 3D datasets. A square region of interest (ROI) was contoured on all included slices within the same XY coordinates, and then interpolated between adjacent slices into a volume of interest (VOI) representing the whole sample model. The cuboid overall VOI with a final dimension of $1072 \times 1072 \times 100$ voxels was thus virtually defined, containing smooth and coarse surfaces. Then, the overall VOI was horizontally hemi-sectioned into the upper and lower VOIs with isometric base area, height, and volume $(1072 \times 1072 \times 50$ voxels $)$, containing smooth and coarse surfaces on each. These VOIs were designated as overall, smooth and coarse ones, respectively (Fig. 1). The upper and lower VOIs were termed as smooth and coarse layers, respectively.

All the 3D images were first preprocessed to reduce noise and enhance contrast using a $3 \times 3 \times 3$ Gaussian filter, with the width and support set at 0.8 and 1.0, respectively. To segment solid polyethylene matrix from pores, global thresholding was implemented with optimal threshold set at 31/137 after repeated trials by combining image histogram and visual estimation. The 3D microstructural analysis was conducted with direct 3D methods on a basis of no structure model assumption using a specialized bone trabecular morphometry algorithm for all the VOIs. The object surface and volume were calculated from the surface meshes generated by surface triangularization language using a specific smoothing parameter. The average pore diameter and strut thickness, equivalent to trabecular separation and thickness in trabeculae, were directly measured by, respectively, fitting maximal spheres into pores and polyethylene struts as described ${ }^{23}$. The Conn.D and DA were also directly calculated. The specific surface area was determined as the ratio of solid matrix surface (BS) to total apparent volume (TV). And the volume porosity was calculated as the percent of the difference (also pore volume) between TV and solid matrix volume (BV) then divided by TV. They could be concisely expressed as

$$
\begin{aligned}
& \text { Specific surface area }=\frac{\mathrm{BS}}{\mathrm{TV}}, \\
& \text { Volume porosity }(\%)=\frac{\mathrm{TV}-\mathrm{BV}}{\mathrm{TV}} \times 100
\end{aligned}
$$

where all the above abbreviations were consistent with the standardized nomenclature and symbols for bone histomorphometry ${ }^{24}$, and the current guidelines for bone microstructure assessment using micro-CT ${ }^{25}$.

\section{Statistical analysis}

Data analysis was performed with SPSS STATISTICS 19.0 for Windows (SPSS Inc., USA). All the 2D topological and geometric datasets were examined with Kolmogorov-Smirnov test, validated as skewed, and thus tested with Mann-Whitney U test. The 2D pore amount, pore density and surface porosity, and all six 3D microstructural datasets were examined with Shapiro-Wilks test. They were all validated as normally distributed, and tested with independent sample $t$ test. Significance level (alpha) was set at 0.05 .

\section{RESULTS}

\section{D pore characterization}

\section{Topological characteristics}

Eligible pores on two surfaces were successfully identified, segmented, and best fitted with ellipses, and displayed at two pore area ranges in Fig. 2. The 2D topological and geometric features of pores appeared different on two surfaces: the smooth surface presented sparse isolated or less-interconnected polygonal pores among major continuous thick solid polyethylene struts on a relatively flat plane, whereas the coarse surface demonstrated tremendous open wellinterconnected polygonal pores among minor thin struts on a rugged plane. On some of the pore walls of two surfaces, especially on coarse ones, one 

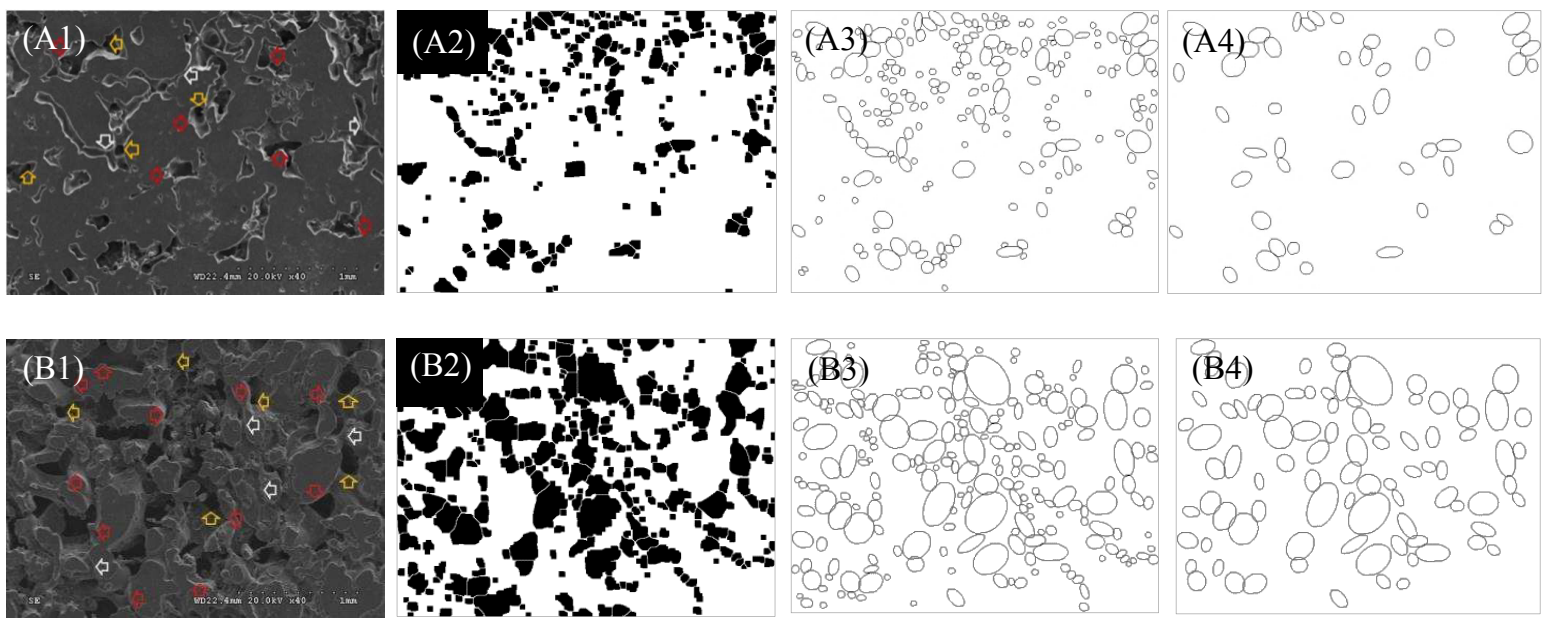

Fig. 2 Representative SEM micrographs (A1, B1) original magnification $\times 40$, segmented pores (A2, B2), best-fitting ellipses with pore area ranging $0 \mu \mathrm{m}^{2}$ - infinity (A3, B3) and $7850 \mu \mathrm{m}^{2}$ - infinity (A4, B4) of smooth (A) and coarse (B) surfaces of PPE membranes within IMAGEJ. The arrows in red, yellow and white indicated typical pores, pore openings and interconnections, respectively.

or multiple circular or quasi-circular pore openings could be found extending from one pore and interconnected with adjacent pores.

\section{Pore size distribution}

The pore size distribution calculated as pore area and circle diameter on two surfaces in two pore area ranges is shown in Fig. 3. With pore area ranging $0 \mu \mathrm{m}^{2}$ - infinity, the vast majority of eligible pores on two surfaces clustered at a pore area range of 0-30000 $\mu \mathrm{m}^{2}$ and a corresponding circle diameter range of $50-200 \mu \mathrm{m}$. With pore area ranging 7850 $\mu \mathrm{m}^{2}$ - infinity, the absolute numbers of remaining pores on two surfaces dramatically decreased. The pore area and circle diameter mainly lied within $7850-50000 \mu \mathrm{m}^{2}$ and $100-250 \mu \mathrm{m}$, respectively.

\section{Topological and geometric quantification}

The results of all the topological and geometric parameters of pores on smooth and coarse surfaces at two area ranges were summarized in Table 1. With pore area ranging $0 \mu \mathrm{m}^{2}-$ infinity, the total amounts of eligible pores on nine images of smooth and coarse surfaces were 1644 and 2211, respectively. There were significant differences in all seven topological and geometric parameters between two surfaces $(p<0.05)$. The smooth surfaces had significantly smaller median pore area, circle diameter, Feret diameter and aspect ratio, but larger roundness, circularity and solidity than the coarse
Table $12 \mathrm{D}$ topological and geometric characteristics of pores of two surfaces at two area ranges $(n=9)$ !

\begin{tabular}{|c|c|c|c|c|c|}
\hline Area range & Parameter & Surface & Mean & SD & CV \\
\hline \multirow{14}{*}{$\begin{array}{l}0 \text { - infinity } \\
\left(\mu \mathrm{m}^{2}\right)\end{array}$} & \multirow{6}{*}{$\begin{array}{l}\text { Pore area* } \\
\left(\times 10^{3} \mu \mathrm{m}^{2}\right) \\
\text { Circle diameter* } \\
(\mu \mathrm{m}) \\
\text { Feret diameter* } \\
(\mu \mathrm{m})\end{array}$} & Smooth & 6.8 & 7.7 & 113.0 \\
\hline & & Coarse & 10.3 & 12.5 & 120.9 \\
\hline & & Smooth & 84.8 & 39.0 & 46.0 \\
\hline & & Coarse & 103.7 & 49.2 & 47.4 \\
\hline & & Smooth & 111.7 & 55.3 & 49. \\
\hline & & Coarse & 136.1 & 67.0 & 49. \\
\hline & \multirow{2}{*}{ Roundness } & Smooth & 0.79 & 0.15 & $18.3^{3}+x$ \\
\hline & & Coarse & 0.78 & 0.14 & 17.6 \\
\hline & \multirow{2}{*}{$\underset{*}{\text { Circularity }}$} & Smooth & 0.83 & 0.08 & 10.1 \\
\hline & & Coarse & 0.81 & 0.09 & 10.7 \\
\hline & \multirow{2}{*}{ Solidity } & Smooth & 0.95 & 0.05 & 4.7 \\
\hline & & Coarse & 0.94 & 0.05 & \\
\hline & \multirow{2}{*}{ spect ratio } & Smooth & 1.31 & 0.29 & 22.3 \\
\hline & & Coarse & 1.33 & 0.28 & 20.9 \\
\hline \multirow{14}{*}{$\begin{array}{l}7850 \text { - infinity } \\
\left(\mu \mathrm{m}^{2}\right)\end{array}$} & \multirow{2}{*}{$\begin{array}{l}\text { Pore area } \\
\left(\times 10^{3} \mu \mathrm{m}^{2}\right)\end{array}$} & Smooth & 16.7 & 10.4 & 62.2 \\
\hline & & Coarse & 19.3 & 16.2 & 83. \\
\hline & \multirow{2}{*}{$\begin{array}{l}\text { Circle diameter } \\
(\mu \mathrm{m})\end{array}$} & Smooth & 140.9 & 37.5 & 26. \\
\hline & & & 148.5 & 51.2 & 34. \\
\hline & \multirow{2}{*}{$\begin{array}{l}\text { Feret diameter } \\
(\mu \mathrm{m})\end{array}$} & Smooth & 190.0 & 55.6 & 29.3 \\
\hline & & Coarse & 196.8 & 70.3 & 35. \\
\hline & \multirow{2}{*}{ Roundness } & Smooth & 0.68 & 0.13 & 19.3 \\
\hline & & Coarse & 0.71 & 0.13 & 18.5 \\
\hline & \multirow{2}{*}{ Circularity } & Smooth & 0.73 & 0.09 & 12. \\
\hline & & Coarse & 0.74 & 0.09 & 12. \\
\hline & \multirow{2}{*}{ Solidity } & Smooth & 0.90 & 0.04 & 4. \\
\hline & & & 0.91 & 0.04 & \\
\hline & \multirow[t]{2}{*}{ Aspect ratio } & & 1.54 & 0.34 & 22.3 \\
\hline & & Coarse & 1.46 & 0.31 & 21. \\
\hline
\end{tabular}

$\dagger \mathrm{SD}=$ standard deviation; $\mathrm{CV}=(\%)$ coefficient of variance; " statistically significant between two surfaces $(p<0.05)$, two-tailed.

surfaces.

With pore area ranging $7850 \mu \mathrm{m}^{2}$ - infinity, the pores with an area below $7850 \mu \mathrm{m}^{2}$ were excluded. The total amounts of eligible pores on smooth and 

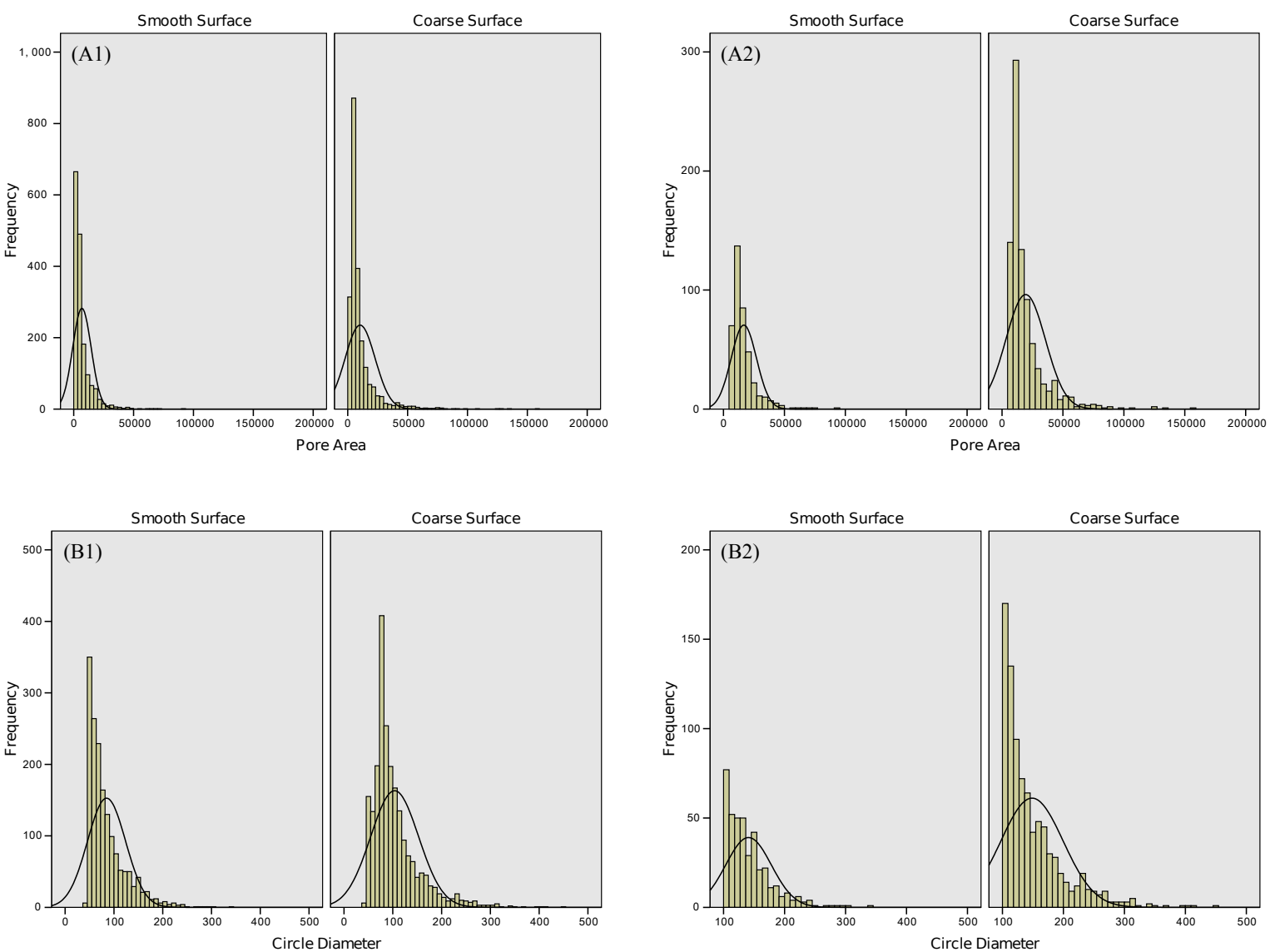

Fig. 3 Pore size distribution histograms in pore area (A) and circle diameter (B) on two surfaces of PPE membranes with pore area ranging $0 \mu \mathrm{m}^{2}$ - infinity (A1, B1) and $7850 \mu \mathrm{m}^{2}$ - infinity (A2, B2). The bar height indicated absolute number of pores, whose size lied between the range on the horizontal axis.

coarse surfaces dropped down to 404 and 862, respectively. No significant differences in median pore area, circle diameter and Feret diameter were found between two surfaces $(p>0.05)$. There were significant differences in the median pore roundness, circularity, solidity and aspect ratio between two surfaces $(p<0.05)$. The smooth surfaces possessed significantly smaller pore roundness, circularity, solidity, but larger aspect ratio than the coarse surfaces.

The pore amount, pore density and surface porosity of two surfaces at two area ranges were presented in Table 2. Significant differences in all three parameters were found between two surfaces at two area ranges $(p<0.05)$. The smooth surfaces had significantly smaller mean pore amount, pore density and surface porosity than the coarse surfaces at two area ranges.

www.scienceasia.org
Table 2 Pore amount, pore density and surface porosity evaluated on two surfaces at two area ranges $(n=9)$ !

\begin{tabular}{|c|c|c|c|c|c|}
\hline Area Range & Parameter & Surface & Mean & SD & $\mathrm{CV}$ \\
\hline \multirow{6}{*}{$\begin{array}{l}0 \text { - infinity } \\
\left(\mu \mathrm{m}^{2}\right)\end{array}$} & \multirow{2}{*}{$\begin{array}{l}\text { Pore Amount } \\
\text { (n) }\end{array}$} & Smooth & 182.7 & 58.8 & 32.2 \\
\hline & & & 245.7 & 47.1 & 19.2 \\
\hline & \multirow{3}{*}{$\begin{array}{l}\text { Pore Density } \\
\left(\times 10^{-5}{\left.\text { per } \mu \mathrm{m}^{2}\right)}\right) \\
\text { Surface Porosity }\end{array}$} & Smooth & 2.4 & 0.8 & 32.4 \\
\hline & & Coarse & 3.2 & 0.6 & 19.1 \\
\hline & & Smooth & 16.3 & 3.8 & 23.3 \\
\hline & $(\%)$ & Coarse & 33.1 & 4.1 & 12.3 \\
\hline \multirow{6}{*}{$\begin{array}{l}7850-\text { infinity } \\
\left(\mu \mathrm{m}^{2}\right)\end{array}$} & \multirow{2}{*}{$\begin{array}{l}\text { Pore Amount } \\
\text { (n) }\end{array}$} & Smooth & 44.9 & 10.6 & 23.6 \\
\hline & & & 95.8 & 7.8 & 8.1 \\
\hline & \multirow{3}{*}{$\begin{array}{l}\text { Pore Density" } \\
\left(\times 10^{-5} \text { per } \mu m^{2}\right) \\
\text { Surface Porosity }\end{array}$} & Smooth & 0.6 & 0.1 & 24.1 \\
\hline & & Coarse & 1.3 & 0.1 & 8.0 \\
\hline & & & 9.8 & 2.5 & 25.9 \\
\hline & (\%) & Coarse & 24.1 & 4.6 & 18.8 \\
\hline
\end{tabular}

${ }^{\dagger} \mathrm{SD}=$ standard deviation; $\mathrm{CV}=(\%)$ coefficient of variance; " statistically significant between two surfaces $(p<0.05)$, two-tailed.

\section{D pore characterization}

\section{Microstructural characteristics}

The density of solid polyethylene matrix was much higher than that of air (pores) in 2D projections, 

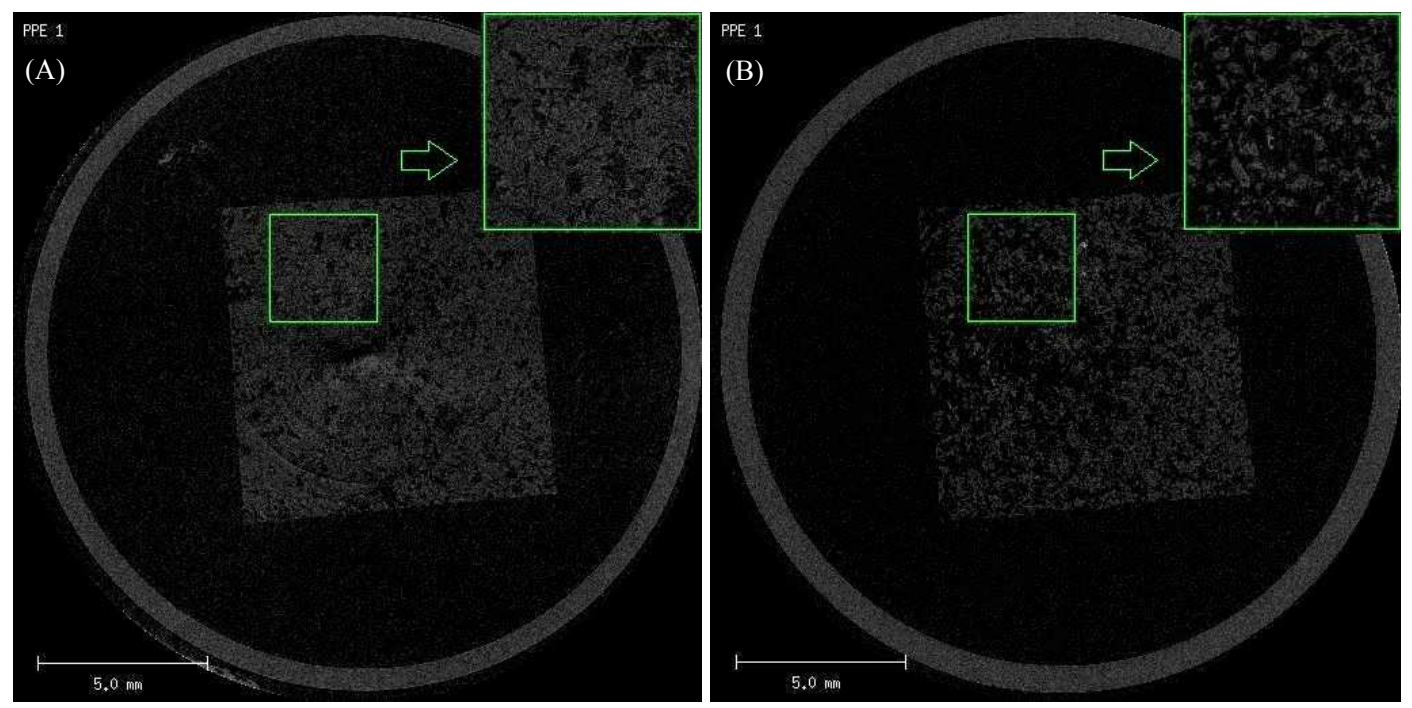

Fig. 4 Representative 2D axial tomographic projections from the smooth (A) and coarse (B) layers of PPE membrane. Green squares outlined the ROIs. The differential content and distribution of polyethylene struts and pores were evident in the zoomed ROIs on the upper right corner. Scale bar $=5.0 \mathrm{~mm}$.

which made it possible to identify and segment polyethylene matrix from pores. The content and distribution of both polyethylene matrix struts and pores appeared quite different on two layers: the polyethylene matrix struts was more compact on smooth layers than on coarse ones. The pores were smaller and more homogeneous on smooth layers than on coarse ones (Fig. 4).

With the aid of Scanco 3D image analysis software, all the PPE membrane models could be virtually segmented into two independent but complementary 3D image datasets for polyethylene matrix and pores, respectively. To quantify the dimension of polyethylene struts and pores, the greyscale segmented 3D images (Figs. 5 and 6: A1, A2, A4, A6, B1, B2, B4, and B6) were converted into the thickness maps and separation maps (Figs. 5 and 6: A3, A5, B3, and B5).

In both segmented images and thickness maps of polyethylene matrix, the superficial struts were indicated as high brightness area, whereas the pores were inversely hidden as low brightness area. The smooth layer mainly consisted of bright compact strut networks, homogeneous fine less-interconnected pores within the models, and few large depression-like pore artefacts across the model boundaries. The coarse layer consisted of sparser strut networks, fewer homogeneous fine well-interconnected pores within the models, and increasing evenly-distributed large depression-like pore artefacts across the model boundaries. Large pore artefacts could penetrate into various depths, and could even perforate through the entire layer, thus forming the perforating throats across the models. This was especially prominent in the coarse layers. The thickness maps indicated that the maximum strut thicknesses in smooth layer (range 48$72 \mu \mathrm{m}$ ) were generally larger than those in coarse layer (range 42-54 $\mu \mathrm{m}$ ) in all three samples (not all data shown). The distribution of strut dimension was generally homogeneous in both layers (Fig. 5).

In contrast, the superficial pores and pore artefacts were indicated as high brightness area, whereas the polyethylene struts were hidden as low brightness area in the segmented pore images and separation maps. The smooth layer mainly consisted of well-interconnected struts and pores, and several large isolated pore artefacts, while the coarse layer mainly consisted of well-interconnected pores and pore artefacts, and sparse struts. The fine pores became sparser; and the large pore artefacts became larger and more intensive from the smooth layer to the coarse layer. Occasionally, a few small perforating channels could be observed in the top and upwards views of two layers, indicating the presence of trans-membrane straight struts perpendicular to membrane surfaces. The separation maps revealed that the maximum pore diameters in smooth layer (range 51-66 $\mu \mathrm{m}$ ) were generally smaller than those in coarse one (range 72-81 $\mu \mathrm{m}$ ) (not all data shown). However, the pore diameter distribution was heterogeneous across the 

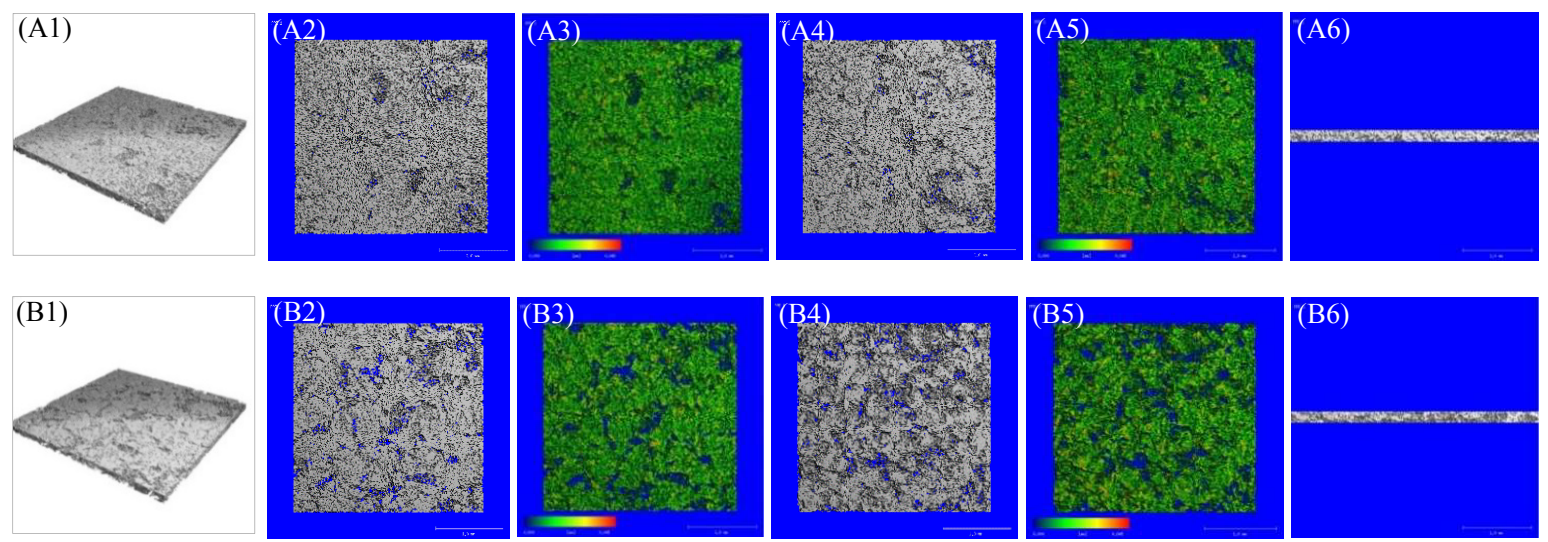

Fig. 5 Representative 3D segmented images and thickness maps of polyethylene matrix of smooth (A) and coarse (B) layers at different views: (A1, B1) oblique top view of two layers, (A2, B2) top view of two layers, (A3, B3) top view of thickness maps of two layers, (A4, B4) upwards view of two layers, (A5, B5) upwards view of thickness maps of two layers, and (A6, B6) cross-sectional view of two layers. The low-brightness depressions on both surfaces outlined typical pore artefacts and perforating throats. Scale bar $=1.0 \mathrm{~mm}$.
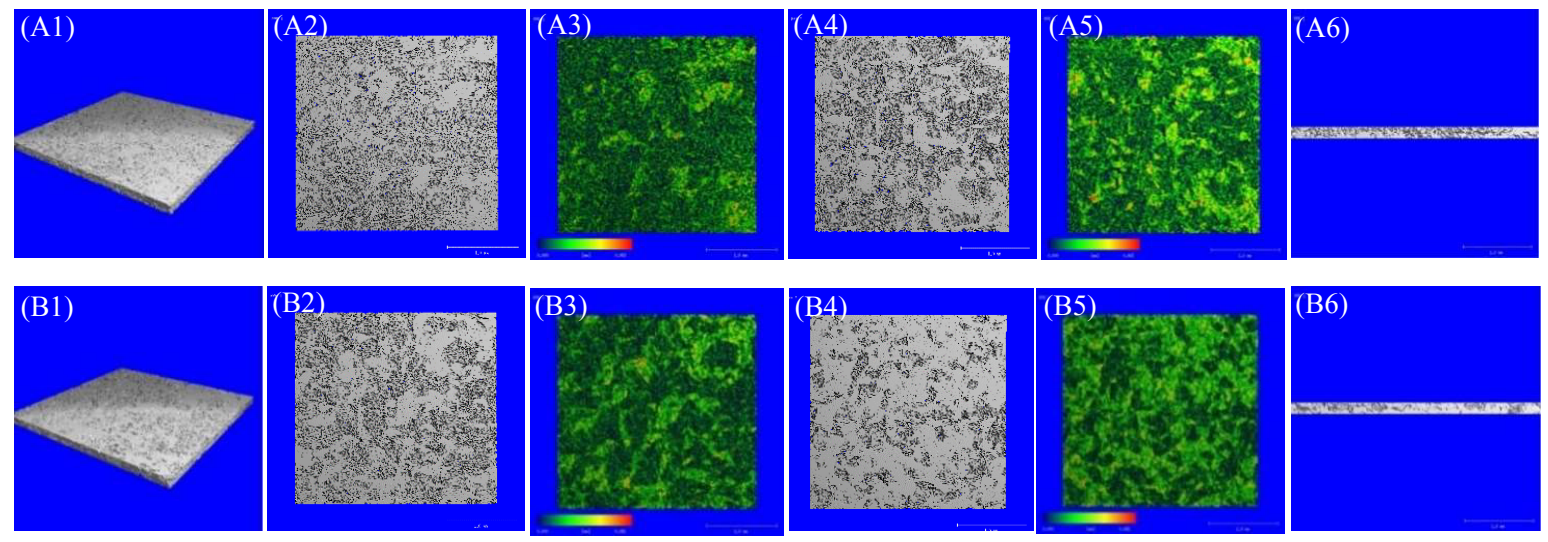

Fig. 6 Representative 3D segmented pores and separation maps of smooth (A) and coarse (B) layers at different views: (A1, B1) oblique top view of two layers, (A2, B2) top view of two layers, (A3, B3) top view of separation maps of two layers, (A4, B4) upwards view of two layers, (A5, B5) upwards view of separation maps of two layers, and (A6, B6) cross-sectional view of two layers. The blue spiculate spots through both surfaces indicated typical trans-membrane straight struts. Scale bar $=1.0 \mathrm{~mm}$.

membrane (Fig. 6).

\section{Morphometric quantification}

The results of six 3D microstructural parameters of two membrane layers were summarized in Table 3. There were significant differences in pore diameter and volume porosity between two layers $(p<0.05)$. The smooth layers had significantly smaller pore diameter and volume porosity than the coarse layers. However, no significant differences in strut thickness, specific surface area, Conn.D and DA were found between two layers $(p>0.05)$.

\section{DISCUSSION}

The combination of SEM images and image analysis made the quantification of 2D pore size, pore size distribution, and pore geometry possible and efficient. 2D pore characterization by using SEM images could only provide two-dimensional greyscale image, partially show the internal sample structure but not completely sections, and thus might underestimate the pore size ${ }^{26}$. Various image analysis programs may have inconsistent results in quantifying image features for various reasons, such as different programming languages, theoretical formulae, algorithmic implementations, input parame- 
Table 3 3D microstructural parameters of both layers of PPE membranes measured by micro-CT $(n=3)$ !

\begin{tabular}{llcrr}
\hline Parameter & Layer & Mean & SD & CV \\
\hline Pore diameter & Smooth & 21.6 & 1.6 & 7.4 \\
$(\mu \mathrm{m})$ & Coarse & 30.0 & 1.4 & 4.7 \\
Volume porosity $^{*}$ & Smooth & 41.3 & 7.5 & 18.2 \\
$(\%)$ & Coarse & 61.0 & 2.5 & 4.1 \\
Strut thickness & Smooth & 24.7 & 5.1 & 20.6 \\
$(\mu \mathrm{m})$ & Coarse & 20.4 & 1.3 & 6.4 \\
Specific surface area & Smooth & 50.9 & 6.6 & 13.0 \\
$\left(\mathrm{~mm}^{2} / \mathrm{mm}^{3}\right)$ & Coarse & 46.9 & 0.4 & 0.9 \\
Conn.D & Smooth & 21.8 & 7.3 & 33.5 \\
$\left(\times 10^{3} \mathrm{~mm}^{-3}\right)$ & Coarse & 19.5 & 1.4 & 7.4 \\
DA & Smooth & 1.2 & 0.1 & 10.7 \\
& Coarse & 1.1 & 0.0 & 2.7 \\
\hline
\end{tabular}

$\dagger \mathrm{SD}=$ standard deviation; $\mathrm{CV}=$ (\%) coefficient of variance; Conn. $\mathrm{D}=$ connectivity density; $\mathrm{DA}=$ degree of anisotropy; * statistically significant between two layers $(p<0.05)$, two-tailed.

ters, measurement units, and ROI definitions, etc ${ }^{27}$. Inappropriate image quality and magnification, and thresholding subjectivity, could also affect the result of image analysis ${ }^{8,28}$. Poor image quality could affect the accurate segmentation for subtle intensity difference of emitted secondary electrons between pores and background. Too low image magnification may lead to the insufficient contrast between the tiny shallow pores and the background. The tiny pores are prone to being omitted during segmentation. In this study, the vast majority of pores imaged under a $\times 40$ magnification had been successfully identified and included for analysis. Further, no matter which segmentation technique is adopted, local or global thresholding techniques, human factors are always involved, thus making the estimation bias inevitable. In addition, the small area captured for analysis and the incapability for pore interconnectivity analysis, may also limit its wide application ${ }^{8}$.

Based on the internal features of IMAGEJ, a purposive cut-off value for some specific parameters (e.g., pore area, and circularity in this study), can be selected for pore analysis. The shown form, result display, considerations for holes and pores on the edges, and others, can be defined as well for specific purposes. In this study, a cut-off pore area of 7850 $\mu \mathrm{m}^{2}$, corresponding to a circle diameter of $100 \mu \mathrm{m}$, was intentionally set. Thus, two ranges of pore area $\left(0 \mu \mathrm{m}^{2}-\right.$ infinity and $7850 \mu \mathrm{m}^{2}-$ infinity $)$ were chosen for parallel analysis. This practice had the following vital considerations and significances.
Firstly, when a significant cut-off pore area value $\left(7850 \mu \mathrm{m}^{2}\right)$ was set, it would facilitate the topological and geometric assessments of pores with area under and above $7850 \mu \mathrm{m}^{2}$. The full area range $(0$ $\mu \mathrm{m}^{2}$ - infinity) could include all the pores on two surfaces for overall analysis, while the partial area ranges $\left(0 \mu \mathrm{m}^{2}-7850 \mu \mathrm{m}^{2}\right.$ and $7850 \mu \mathrm{m}^{2}$ - infinity) could facilitate clarifying two specific groups of eligible pores for specific purposes.

Secondly, though the minimum and optimal pore sizes for bone ingrowth remain highly controversial, it was reported that the pore size over $100 \mu \mathrm{m}$ could facilitate the rapid ingrowth of wellvascularized connective tissue and then direct osteogenesis, while the one under $100 \mu \mathrm{m}$ tended to be penetrated with more avascular connective tissue, thus leading to osteochondral formation for hypoxic conditions ${ }^{29,30}$. The macro pores were more favourable for in vivo cell adhesion, migration, differentiation, neovascularization, and new bone formation than the micro ones ${ }^{30-32}$. Furthermore, the optimal bone ingrowth was found in pores ranging 100-135 $\mu \mathrm{m}$, though bone could grow into the small pores of porous polyethylene down to $40 \mu \mathrm{m}^{16}$. The PSD in this study revealed that the vast majority of pores on two surfaces of PPE membrane had a circle diameter ranging 50$200 \mu \mathrm{m}$. Thus all of them might enable the bone ingrowth. Furthermore, when the circle diameter was set above $100 \mu \mathrm{m}$, only $25 \%$ and $39 \%$ of all the pores on the smooth and coarse surfaces could be designated as eligible, respectively. The median and mean values on smooth and coarse surfaces could reach up to $130 \mu \mathrm{m}$ and $140 \mu \mathrm{m}$, respectively. The macro pores (over $100 \mu \mathrm{m}$ ) on both surfaces, especially the coarse ones, could enable optimal bone ingrowth, whereas the micro pores (under $100 \mu \mathrm{m}$ ) on both surfaces, especially the smooth ones, might be more prone to being occluded by the fast-growing epithelial cells and avascular soft tissue, and thus leading to less or no bone formation.

Thirdly, though an optimal pore size has not yet been determined for barrier membrane until today $^{31}$, it was believed that, the macroporous membranes with pore size ranging 100-325 $\mu \mathrm{m}$ could well balance the pore size, porosity, mechanical properties, and biological responses compared with the microporous ones ${ }^{30}$. In this study, of all the pores on the smooth and coarse surfaces, approximately $75 \%$ and $61 \%$ were the micro ones with a circle diameter under $100 \mu \mathrm{m}$ as revealed in Table 2. They would enable the early ingrowth of a small quantity of fast-growing epithelial cells 
and avascular soft tissue for postoperative tissue colonization and membrane stabilization. Then, these pre-colonizing cells and tissues would probably occlude the micro pores, and thus efficiently inhibit further penetration of fibroblasts and soft tissue from the mucoperiosteal flap due to the cellular growth inhibition effect. The pore size and PSD characteristics could simultaneously endow the property of cell occlusiveness and abdicate the space beneath membranes for bone regeneration. Hence the selection of the cut-off values for pore area $\left(7850 \mu \mathrm{m}^{2}\right)$ and circle diameter $(100 \mu \mathrm{m})$ was reasonable and appropriate in this study.

In addition, the pore geometry of porous scaffolds and biomaterials has been validated to significantly influence cellular response and tissue ingrowth, and to regulate the organization and orientation of collagen fibres, thus remarkably affecting the tissue structure and mechanics. The widelyaccepted curvature-driven tissue growth model indicates that, all the angiogenesis, tissue growth, and bone formation and mineralization, prefer a higher pore curvature in porous scaffolds, and tissue generation is much more on concave surfaces than convex and planar ones ${ }^{33,34}$. A higher roundness, circularity, solidity and lower aspect ratio of 2D pores indicate a higher pore curvature and more concave surface, while a lower roundness, circularity, solidity and higher aspect ratio indicate a lower curvature and more convex/planar surface. Based on this knowledge, the results of pore geometric parameters (i.e., roundness, circularity, solidity and aspect ratio) in this study might imply that, the predominant fibroblasts and soft tissue from mucoperiosteal prefer the overall pores $\left(0 \mu \mathrm{m}^{2}-\right.$ infinity) on smooth surfaces to those on coarse surface, thus leading to quick pore occlusion, whereas the overwhelming bone-forming cells and bone tissue from medullary space prefer the macro pores (7850 $\mu \mathrm{m}^{2}$ - infinity) on coarse surfaces to those on smooth surfaces, thus leading to more bone formation and ingrowth. Hence the design of differential pore geometries on both surfaces was speculated to contribute to the cell occlusiveness on smooth surfaces and to enhance the bone regeneration on coarse surfaces. Furthermore, these macro pores might be coated by plasma proteins upon implantation, thus facilitating the cellular adhesion and hermetic seal formation ${ }^{34}$. The mechanism might provide additional resistance to the migration of epithelial cells and the invasion of oral microbes when exposed. However, these speculations remain to be verified. To acquire so precisely-controlled pore geometry gradients on such a thin membrane, additive manufacturing techniques (e.g., 3D printing) possess unrivalled advantages ${ }^{33,34}$, and will be one of our future research orientations.

From 2D analysis, bi-layered PPE membrane possessed different pore sizes, PSDs, geometries, pore amounts, pore densities, and surface porosities on two surfaces. All these unique 2D pore structures could synergistically contribute to the cell occlusiveness on smooth surface and the bone regeneration potential on coarse surface.

In contrast to 2D analysis, micro-CT could enable simultaneous 2D and 3D characterizations of the internal microstructures of bi-layered PPE membranes. It could separate two independent but complementary 3D datasets for polyethylene matrix and pores with associated software. Two segmented components could be transformed into coloured thickness and separation maps, which could intuitively illustrate the component size and distribution. Their microstructures could be quantified in a direct 3D manner without any model assumption using multiple image analysis languages (e.g., distance transformation, surface triangularization, and mean intercept length) ${ }^{35}$. The use of submicronlevel resolution could ensure its high accuracy ${ }^{8-10}$. However, it required huge investments on equipment procurement and maintenance, and extensive personnel trainings to fully use the advanced functions and obtain the reliable outcomes ${ }^{8}$.

From morphometric quantification, the mean pore diameters of both layers of PPE membrane measured by micro-CT were quite small, ranging 20-30 $\mu \mathrm{m}$, compared to the values obtained by 2D analysis. Most of current micro-CT systems, including Scanco system, were originally developed for bone morphometry which mainly characterized trabecular microstructures ${ }^{25}$. Porous material structures, however, come in various natures due to different fabrication techniques or processes and the shape of the pores could be far different from those of trabeculae which were rather isotropic. The use of the best-fitting sphere in the micro-CT analysis algorithm underestimated the pore size, since it isotropically measured the pores by fitting the largest sphere but discarded the long-axis dimension of anisotropic pores. The use of separation map could provide all the data of individual spheres which were fitted in the pores for the membrane for additional analysis. The maximum pore diameters (51-66 $\mu \mathrm{m}$ and $72-81 \mu \mathrm{m}$ for smooth and coarse layers, respectively) in the separation maps were found to be greater than the values 
from morphometric quantification data and closer to those of 2D analysis. As with 2D analysis, high volume porosities were also found in both layers and the porosity was greater in the coarse layers than in the smooth ones. This would be beneficial and desirable for providing high permeability for nutrient, oxygen and metabolite diffusions and new bone formation ${ }^{30}$. As all the pores, pore artefacts and perforating throats would serve as space for cell and tissue ingrowths, they were all included for 3D morphometric analysis within the internal features of micro-CT. The smooth layer possessed fewer large pores, pore artefacts and perforating throats than the coarse one, thus contributing to the significantly smaller pore diameter and volume porosity.

On the other hand, the nonsignificant difference in strut thickness confirmed the homogeneity of polyethylene struts across the membrane. Combined with the significantly different pore diameters and volume porosities, this feature might contribute to the homogeneous polyethylene matrix structure, facilitate the intraoperative membrane contouring towards coarse layer (also bone defect side), and maintain the structural integrity in clinical application $^{30}$. Furthermore, the specific surface area and Conn.D of both layers were much higher than those of healthy human cancellous bone taken from tibial condyles (mean $3.95 \mathrm{~mm}^{2} / \mathrm{mm}^{3}$, range 2.09 $5.42 \mathrm{~mm}^{2} / \mathrm{mm}^{3}$, and mean $6.86 \mathrm{~mm}^{-3}$, range 2.55 $12.8 \mathrm{~mm}^{-3}$, respectively) ${ }^{36,37}$. According to the biomimetic design principle for biomaterials and scaffolds, high specific surface area was favourable for initial cell attachment, migration and osteoblast proliferation $^{32,38}$, while high Conn.D values indicated the extremely high degrees to which the polyethylene strut network on both layers was interconnected, and large maximum numbers of matrix connections that were needed to be broken before dividing into two parts $25,36,39$. Its nonsignificant difference might contribute to the consistent mechanical property and space maintenance across the entire membrane. Finally, the DAs of both layers were approximately 1.0, and not significantly different, implying similar and isotropic matrix structure across the membrane ${ }^{25}$.

In comparison to commercial expanded/dense polytetrafluoroethylene membranes which, respectively, had the pore sizes of $8 \mu \mathrm{m}$ and $0.3 \mu \mathrm{m}$ which were claimed to be able to partially retard or completely block the invasion of individual oral microbes in case of premature wound dehiscence and membrane exposure ${ }^{40}$, the pore sizes of PPE membrane were still larger. However, due to the complex membrane microstructures, cell and tissue behaviours within porous biomaterials and microbehost interactions, whether the bi-layered PPE membrane possesses the bacterium occlusiveness remains to be confirmed.

SEM and micro-CT were two of the most commonly-used techniques for microstructural characterization. They had their own advantages and limitations. In this study, these two techniques had been successfully applied in characterizing the newly-developed bi-layered PPE membrane. Their combination was found to compensate their mutual limitations and synergistically elucidate the pore microstructures of bi-layered PPE membrane.

\section{CONCLUSIONS}

The 2D and 3D pore characteristics as revealed from the SEM and micro-CT image analyses could be complementarily employed to characterize the pore microstructure and morphology of PPE barrier membrane. A developed PPE membrane was proved to comprise bi-layered structure having low porosity on one side and high porosity on the other side as designed. In the future, the mechanical and biological property measurements, for example, tensile properties, cell proliferation, and cell / bacterium occlusiveness, are planned to further investigate the suitability of this membrane for using as a membrane in GBR.

Acknowledgements: This work was jointly supported by the 30th Anniversary Faculty of Dentistry Khon Kaen University Research Funding (DTR6001), and the Khon Kaen University Graduate School Thesis Support Scholarship (No. 59212101), and the Neuroscience Research and Development Group, Khon Kaen University, Thailand. The authors acknowledge the Biomaterial Research Laboratory, Faculty of Dentistry, Khon Kaen University and the Oral Biology Research Center, Faculty of Dentistry, Chulalongkorn University, Thailand, for facility support. We gratefully thank Sathaban Sommitr and Sureerat Luangworakhun for their kind assistance in sample imaging. We also appreciate Dr Stephen Weiss at Scanco Medical, Switzerland for his great assistance in software extension and analysis guidance.

\section{REFERENCES}

1. Canger EM,Çelenk P (2012) Radiographic evaluation of alveolar ridge heights of dentate and edentulous patients: residual ridge resorption. Gerodontology 29, 17-23. 
2. Cawood JI, Howell RA (1988) A classification of the edentulous jaws. Int $J$ Oral Maxillofac Surg 17, 232-6.

3. Esposito M, Grusovin MG, Coulthard P, Worthington HV (2006) The efficacy of various bone augmentation procedures for dental implants: a Cochrane systematic review of randomized controlled clinical trials. Int J Oral Maxillofac Implants 21, 696-710.

4. Rakhmatia YD, Ayukawa Y, Furuhashi A, Koyano K (2013) Current barrier membranes: titanium mesh and other membranes for guided bone regeneration in dental applications. J Prosthodont Res 57, 3-14.

5. Dimitriou R, Mataliotakis GI, Calori GM, Giannoudis PV (2012) The role of barrier membranes for guided bone regeneration and restoration of large bone defects: current experimental and clinical evidence. BMC Med 10, 81.

6. Yoshimoto I, Sasaki JI, Tsuboi R, Yamaguchi S, Kitagawa H, Imazato S (2018) Development of layered PLGA membranes for periodontal tissue regeneration. Dent Mater 34, 538-50.

7. Loh QL, Choong C (2013) Three-dimensional scaffolds for tissue engineering applications: role of porosity and pore size. Tissue Eng Part B Rev 19, 485-502.

8. Safinia L, Mantalaris A, Bismarck A (2006) Nondestructive technique for the characterization of the pore size distribution of soft porous constructs for tissue engineering. Langmuir 22, 3235-42.

9. Gregor T, Kochová P, Eberlová L, Nedorost L, Prosecká E, Liška V, Mírka H, Kachlík D, et al (2012) Correlating micro-CT imaging with quantitative histology. In: Goswami T (ed) Injury and Skeletal Biomechanics, IntechOpen.

10. Gramanzini M, Gargiulo S, Zarone F, Megna R, Apicella A, Aversa R, Salvatore M, Mancini M, et al (2016) Combined microcomputed tomography, biomechanical and histomorphometric analysis of the peri-implant bone: a pilot study in minipig model. Dent Mater 32, 794-806.

11. Ciocca L, Lesci I, Donati D, Scotti R (2014) Custom made innovative composite scaffold for bone regenerative medicine. Dent Mater 30, e136-7.

12. Hambli R (2013) Micro-CT finite element model and experimental validation of trabecular bone damage and fracture. Bone 56, 363-74.

13. Torcasio A, Zhang X, Van Oosterwyck H, Duyck J, van Lenthe GH (2012) Use of micro-CT-based finite element analysis to accurately quantify peri-implant bone strains: a validation in rat tibiae. Biomech Model Mechanobiol 11, 743-50.

14. Park HK, Dujovny M, Diaz FG, Guthikonda M (2002) Biomechanical properties of high-density polyethylene for pterional prosthesis. Neurol Res 24, 671-6.

15. Li S, Burstein AH (1994) Ultra-high molecular weight polyethylene. The material and its use in total joint implants. J Bone Joint Surg Am 76, 1080-90.
16. Klawitter JJ, Bagwell JG, Weinstein AM, Sauer BW (1976) An evaluation of bone growth into porous high density polyethylene. J Biomed Mater Res 10, 311-23.

17. Fernandez-Bueno I, Di Lauro S, Alvarez I, Lopez JC, Garcia-Gutierrez MT, Fernandez I, Larra E, Pastor JC (2015) Safety and biocompatibility of a new high-density polyethylene-based spherical integrated porous orbital implant: an experimental study in rabbits. J Ophthalmol 2015, 904096.

18. Rai A, Datarkar A, Arora A, Adwani DG (2014) Utility of high density porous polyethylene implants in maxillofacial surgery. J Maxillofac Oral Surg 13, 42-6.

19. Swords GA, Spagnoli DB (2016) Bone Graft Material Containment Structures, US Patent 9433707 B2.

20. Suwanprateeb J, Thammarakcharoen F, Wongsuvan V, Chokevivat W (2012) Development of porous powder printed high density polyethylene for personalized bone implants. J Porous Mater 19, 623-32.

21. Suwanprateeb J, Suvannapruk W, Wasoontararat K, Leelapatranurak K, Wanumkarng N, Sintuwong S (2011) Preparation and comparative study of a new porous polyethylene ocular implant using powder printing technology. $J$ Bioact Compat Polym 26, 317-31.

22. Suwanprateeb J, Thammarakcharoen F, Suvannapruk W (2013) Preparation and characterization of 3D printed porous polyethylene for medical applications by novel wet salt bed technique. Chiang Mai J Sci 41, 200-12.

23. Hildebrand T, Rüegsegger P (1997) A new method for the model-independent assessment of thickness in three-dimensional images. $J$ Microsc 185, 67-75.

24. Dempster DW, Compston JE, Drezner MK, Glorieux FH, Kanis JA, Malluche H, Meunier PJ, Ott SM, et al (2013) Standardized nomenclature, symbols, and units for bone histomorphometry: A 2012 update of the report of the ASBMR Histomorphometry Nomenclature Committee. J Bone Miner Res 28, 2-17.

25. Bouxsein ML, Boyd SK, Christiansen BA, Guldberg RE, Jepsen KJ, Müller R (2010) Guidelines for assessment of bone microstructure in rodents using micro-computed tomography. J Bone Miner Res 25, 1468-86.

26. Lawrence M, Jiang Y (2017) Porosity, pore size distribution, micro-structure. In: Amziane S, Collet F (eds) Bio-aggregates Based Building Materials, RILEM State-of-the-Art Reports 23, Springer, Dordrecht, pp 39-71.

27. Simon M, Chalfoun J, Brady M, Bajcsy P (2016) Do we trust image measurements? Variability, accuracy and traceability of image features. In: 2016 IEEE International Conference on Big Data, Washington DC, USA, pp 1474-82.

28. AlMarzooqi FA, Bilad MR, Mansoor B, Arafat HA (2016) A comparative study of image analysis and 
porometry techniques for characterization of porous membranes. J Mater Sci 51, 2017-32.

29. Chvapil M, Holuša R, Kliment K, Štoll M (1969) Some chemical and biological characteristics of a new collagen-polymer compound material. J Biomed Mater Res 3, 315-32.

30. Karageorgiou V, Kaplan D (2005) Porosity of 3D biomaterial scaffolds and osteogenesis. Biomaterials 26, 5474-91.

31. Gutta R, Baker RA, Bartolucci AA, Louis PJ (2009) Barrier membranes used for ridge augmentation: is there an optimal pore size? J Oral Maxillofac Surg 67, 1218-25.

32. Murphy CM, Haugh MG, O'Brien FJ (2010) The effect of mean pore size on cell attachment, proliferation and migration in collagen-glycosaminoglycan scaffolds for bone tissue engineering. Biomaterials 31, 461-6.

33. Gariboldi MI, Best SM (2015) Effect of ceramic scaffold architectural parameters on biological response. Front Bioeng Biotechnol 3, 151.

34. Zadpoor AA (2015) Bone tissue regeneration: the role of scaffold geometry. Biomater Sci 3, 231-45.

35. Hildebrand T, Laib A, Müller R, Dequeker J, Rüegsegger P (1999) Direct three-dimensional morphometric analysis of human cancellous bone: microstructural data from spine, femur, iliac crest, and calcaneus. $J$ Bone Miner Res 14, 1167-74.

36. Van Cleynenbreugel T, Schrooten J, Van Oosterwyck H, Vander Sloten J (2006) Micro-CT-based screening of biomechanical and structural properties of bone tissue engineering scaffolds. Med Biol Eng Comput 44, 517-25.

37. Ding M, Odgaard A, Linde F, Hvid I (2002) Agerelated variations in the microstructure of human tibial cancellous bone. J Orthop Res 20, 615-21.

38. O’Brien FJ, Harley BA, Yannas IV, Gibson LJ (2005) The effect of pore size on cell adhesion in collagenGAG scaffolds. Biomaterials 26, 433-41.

39. Odgaard A, Gundersen HJ (1993) Quantification of connectivity in cancellous bone, with special emphasis on 3-D reconstructions. Bone 14, 173-82.

40. Joly JC, de Carvalho PFM, da Silva RC (2017) Esthetic Perio-Implantology, 1st edn, Quintessence, Berlin. 